This item was submitted to Loughborough's Research Repository by the author.

Items in Figshare are protected by copyright, with all rights reserved, unless otherwise indicated.

\title{
A deep adaptive framework for robust myoelectric hand movement prediction
}

PLEASE CITE THE PUBLISHED VERSION

https://www.ukras.org/wp-content/uploads/2019/03/UKRAS19-Proceedings-Final.pdf

\section{VERSION}

VoR (Version of Record)

\section{PUBLISHER STATEMENT}

This work is made available according to the conditions of the Creative Commons Attribution-NonCommercialNoDerivatives 4.0 International (CC BY-NC-ND 4.0) licence. Full details of this licence are available at: https://creativecommons.org/licenses/by-nc-nd/4.0/

\section{LICENCE}

CC BY-NC-ND 4.0

\section{REPOSITORY RECORD}

Robinson, Carl Peter, Baihua Li, Qinggang Meng, and Matthew T.G. Pain. 2019. "A Deep Adaptive Framework for Robust Myoelectric Hand Movement Prediction". figshare. https://hdl.handle.net/2134/37188. 


\title{
A Deep Adaptive Framework for Robust Myoelectric Hand Movement Prediction
}

\author{
Carl Peter Robinson, Baihua Li, Qinggang Meng, and Matthew T.G. Pain
}

\begin{abstract}
This work explored the requirements of accurately and reliably predicting user intention using a deep learning methodology when performing fine-grained movements of the human hand. The focus was on combining a feature engineering process with the effective capability of deep learning to further identify salient characteristics from a biological input signal. 3 time domain features (root mean square, waveform length, and slope sign changes) were extracted from the surface electromyography (sEMG) signal of 17 hand and wrist movements performed by 40 subjects. The feature data was mapped to 6 sensor bend resistance readings from a CyberGlove II system, representing the associated hand kinematic data. These sensors were located at specific joints of interest on the human hand (the thumb's metacarpophalangeal joint, the proximal interphalangeal joint of each finger, and the radiocarpal joint of the wrist). All datasets were taken from database 2 of the NinaPro online database repository. A 3-layer long short-term memory model with dropout was developed to predict the 6 glove sensor readings using a corresponding SEMG feature vector as input. Initial results from trials using test data from the 40 subjects produce an average mean squared error of 0.176. This indicates a viable pathway to follow for this prediction method of hand movement data, although further work is needed to optimize the model and to analyze the data with a more detailed set of metrics.
\end{abstract}

\section{INTRODUCTION}

The potential of the electromyography (EMG) signal generated by human muscles when performing activity has been explored in the myoelectric control of prosthetic devices for many decades, particularly in upper limb mechanisms. Major advancements in commercial hardware have led to instruments capable of intricate mechanical actions. Control strategies, however, have been less successful in their evolution, now seen as a bottleneck to providing effective means of daily life activity for amputees. Use of pattern recognition has garnered significant successes in laboratory conditions [1] and gradual translation to viable end product (www.coaptengineering.com). Much of this has been based on the classification methodology, which is still sequential in nature, only offering one hand function at a time and thus contradictory to the requirement for more fluid, natural movement in myoelectric prosthetics [2]. A more promising approach is the employment of a simultaneous and proportional methodology. Here, the objective is to control multiple degrees of freedom (DOFs) using regression, to estimate a continuous output value for each DOF. This establishes a mapping between EMG input and control output,

C. P. Robinson, B. Li, and Q. Meng are with the Computer Science department, Loughborough University, Loughborough, LE11 3TU, UK. Corresponding author: C.P. Robinson; e-mail: c.p.robinson@ lboro.ac.uk). proving more suitable for intuitive control. Deep learning has found many applications when applied to biological data [3]. In general, when applied to the problem of hand gesture recognition, convolutional neural networks $(\mathrm{CNN})$, long short term memory (LSTM), or combinations of the two are employed [4], [5], [6]. These follow the classification methodology however, with [7] providing the only evidence so far of using regression for continuous prediction of hand kinematic data. They use an autoencoder (AE) to map two kinematic signals via nonlinear regression, for controlling two DOFs. This work uses a 3-layer LSTM model to map sEMG data for 17 human hand and wrist movements, taken from 40 subjects. A preprocessing stage where initial sets of features are extracted from the sEMG data, is used to provide LSTM model input. This is then mapped to 6 glove sensor readings representing the corresponding hand kinematic data.

\section{METHOD}

\section{A. Preprocessing}

Data from 40 intact subjects were downloaded from the NinaPro project website (http://ninapro.hevs.ch), specifically Exercise B's 17 hand and wrist movements from Database 2. The data was initially acquired using 12 Delsys Trigno wireless electrodes (Delsys, Inc, www.delsys.com). Eight electrodes were attached around the right forearm, at a fixed distance from the radio-humeral joint, two were fixed to the main activity spots of the anterior and posterior of the forearm, and two more placed on the biceps brachii and triceps brachii. All movements were repeated 6 times consecutively, each lasting approximately 5 seconds, plus a 3 -second rest period where the subject returned their hand to a rest posture with data acquired at a $2 \mathrm{KHz}$ sampling rate [8]. A CyberGlove II (www.cyberglovesystems.com) equipped with 22 sensors located around the joint positions of the fingers and wrist, was used to acquire hand kinematics. The glove uses resistive bend-sensing technology providing an 8 -bit value ( 0 to 255 ) proportional to the bend angle for each sensor. Six sensors were chosen, representing metacarpophalangeal (MCP) and proximal interphalangeal (PIP) joints of interest around the thumb and fingers of the right hand, and additionally the wrist joint (Table I). This enabled sufficient data capture for all hand movements. An in-house MATLAB program separated movement repetitions into a matrix of time-ordered sEMG voltage data from the electrodes. Each movement's data was split such that repetitions $1,3,4$, and 6 were allocated to a

M. T .G. Pain is with the Sport, Exercise, and Health Sciences department, Loughborough University, Loughborough, LE11 3TU, UK 
training set and repetitions 2 and 5 to a test set. Data were normalized to have zero mean and unit standard deviation [8].
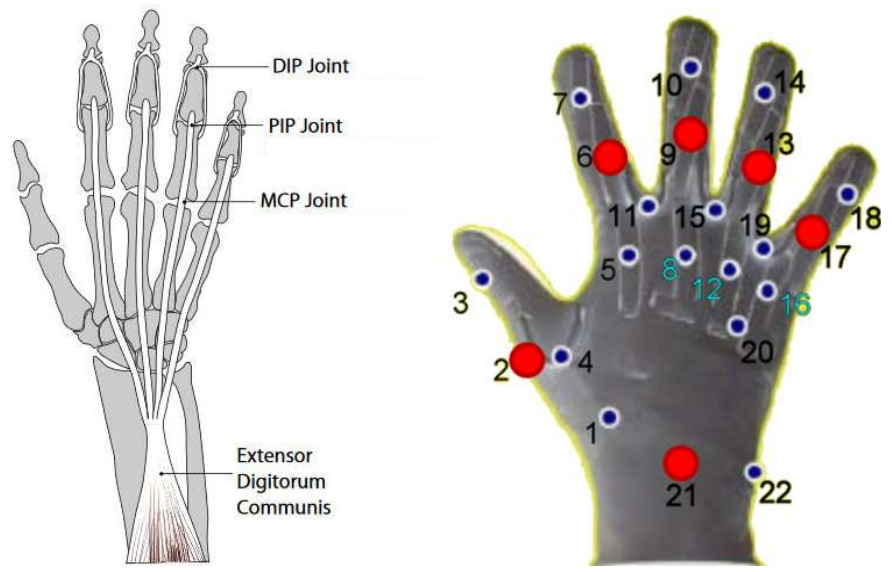

Figure 1. Joints of the finger and CyberGlove sensor locations. Red circles indicate the selected sensors, used for target kinematic data prediction.. Figure created by adapting detail from [9] and [10].

TABLE I. CYBERGLOVE SENSORS OF INTEREST FOR EXPERIMENT

\begin{tabular}{|l|l|}
\hline Sensor & \multicolumn{1}{c|}{ Location } \\
\hline 2 & MCP joint of the thumb \\
\hline 6 & PIP joint of the index finger \\
\hline 9 & PIP joint of the middle finger \\
\hline 13 & PIP joint of the ring finger \\
\hline 17 & PIP joint of the pinky finger \\
\hline 21 & Radiocarpal joint of the wrist \\
\hline
\end{tabular}

A $256 \mathrm{~ms}$ sliding window was employed, as per our previous work, to segment the data. The increment was set at $25 \mathrm{~ms}$ to ensure a densely packed array of windows. Three time domain (TD) features were chosen based on performance in our previous research [11]. The first two are part of the prevalent Hudgins set [12] while the third feature is also commonly used in sEMG research:

- Waveform Length (WL) - a simple rolling calculation of the summation of the absolute difference between a signal sample $x_{k}$ and its previous sample $x_{k-1}$ (equating to $\Delta x_{k}$ ), for all samples $\mathrm{N}$ in one window:

$$
W L=\sum_{k-1}^{N}\left|\Delta x_{k}\right|
$$

- $\quad$ Slope Sign Changes (SSC) - a scalar count in terms of number of times the sEMG signal slope changes between positive and negative values, based on a threshold. Three consecutive window samples are used to check if sample $x_{k}$ is greater or less than its neighbours $\left(x_{k-1}\right.$ and $\left.x_{k+1}\right)$ :

$$
\begin{gathered}
\mathrm{x}_{\mathrm{k}}>\mathrm{x}_{\mathrm{k}-1} \text { and } \mathrm{x}_{\mathrm{k}}>\mathrm{x}_{\mathrm{k}+1} \text {, or } \\
\mathrm{x}_{\mathrm{k}}<\mathrm{x}_{\mathrm{k}-1} \text { and } \mathrm{x}_{\mathrm{k}}<\mathrm{x}_{\mathrm{k}+1} \text {, and } \\
\left|\mathrm{x}_{\mathrm{k}}-\mathrm{x}_{\mathrm{k}-1} \mathrm{x}_{\mathrm{k}+1}\right| \geq 0.01 \mathrm{~V} \text { or }\left|\mathrm{x}_{\mathrm{k}}-\mathrm{x}_{\mathrm{k}-1} \mathrm{x}_{\mathrm{k}-1}\right| \geq 0.01 \mathrm{~V}
\end{gathered}
$$

- $\quad$ Root Mean Square (RMS) - an indicator of average signal value, cancelling out the negative values by squaring them to obtain a mean value:

$$
R M S=\sqrt{\frac{1}{N} \sum_{k=1}^{N} x_{k}^{2}}
$$

Together, these features can provide useful information about sEMG signal waveform complexity and frequency, and muscle contraction intensity. They were extracted from each window, for all 12 electrodes, creating a feature vector $v_{t}$ of 36 scalar features for one time window $t$. Corresponding glove sensor data was windowed using the same procedure and a mean signal feature extracted per window for each sensor. These 6 scalar values were appended to the existing feature vector $v_{t}$. For each hand movement, this process yielded a time-ordered feature matrix $F_{T, E, G}$ consisting of a set of $v_{t}$ feature vectors:

$$
\boldsymbol{F}_{\boldsymbol{T}, \boldsymbol{E}, \boldsymbol{G}}=\left(\begin{array}{ccc}
f_{t, i}^{e} & \cdots & f_{t, i}^{G} \\
\vdots & \ddots & \vdots \\
f_{T, i}^{e} & \cdots & f_{T, i}^{G}
\end{array}\right)
$$

where $e$ is a single sEMG electrode up to a maximum of $E$ electrodes, $i$ represents an extracted feature, $G$ is the total number of glove sensors, and $T$ the total number of time windows in one hand movement.

\section{B. LSTM Model}

An LSTM network is a type of recurrent neural network (RNN) existing as a series of states through time, suitable for analyzing data of a temporal nature, such as the sEMG signal. It utilizes a memory cell consisting of 3 logic gates and a persistent memory state, providing more control than a regular $\mathrm{RNN}$ as to the data flow through the network state per time step [13]. Research into LSTM usage in biosignal fields shows stacking several LSTM layers offers improved model performance [14], capturing more detailed temporal information. Initial trials and related work [15] prompted a design of 3 layers (Fig. 2). The 36 sEMG TD features were used as input to the model, which produced predicted kinematic output in the form of 6 glove sensor values, representative of the corresponding joint movements. The model was configured to evaluate 4 time steps of feature data when making a prediction and each LSTM layer was configured to have 200 hidden nodes, based on a prior optimization investigation that used a subset of subject data. After each layer, a dropout layer was added, implementing an arbitrary dropout rate of $30 \%$ in order to generalize the model and reduce the chance of overfitting. A dense, fully-connected layer using a tanh activation function completed the model, making the actual prediction of the 6 glove sensor outputs. 


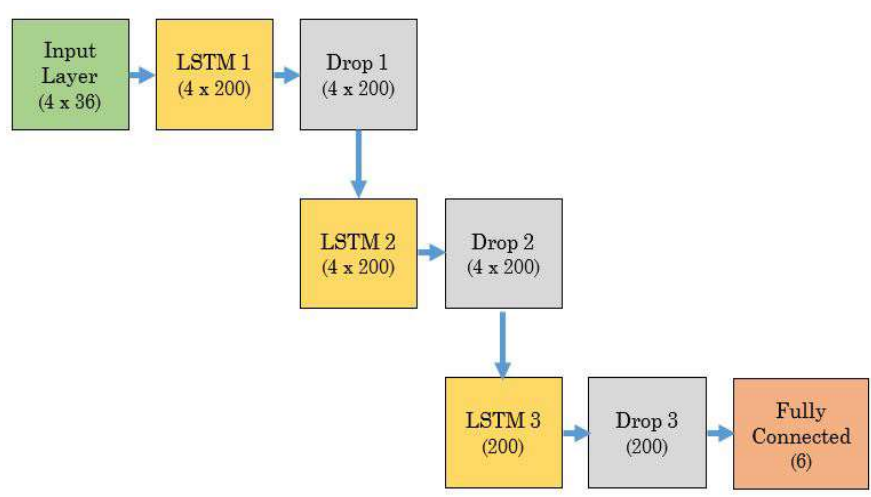

Figure 2. LSTM model structure.

The network was trained using the stochastic gradient descent optimization algorithm, learning rate of 0.3 with momentum of 0.9 applied to the gradient descent operation. Training took place over 40 epochs using a batch size of 600 . All hyperparameters were tuned by conducting an optimization process using a 3 -fold cross validation grid search.

\section{RESULTS}

The mean squared error (MSE) was used as a model performance estimate. Individual subject's test data were evaluated with the trained LSTM model, predicting the 6 DOFs for each hand movement. An average MSE of 0.176 was achieved over 40 subjects and plots indicate reasonable DOF prediction over the 17 hand and wrist movements (Fig. 3).
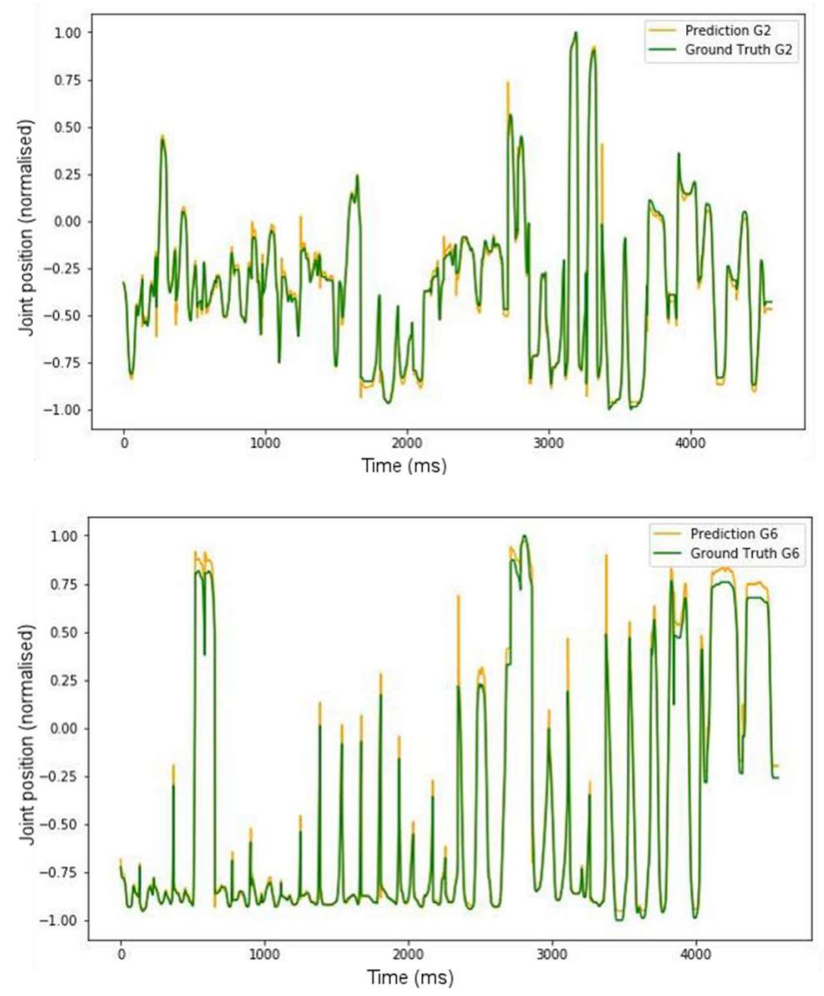

Figure 3. Predicted kinematic data (yellow) against ground truth glove sensor data (green) of thumb MCP joint (top) and index finger PIP joint (bottom) for 17 hand and wrist movements from test dataset of subject 1 .
A more detailed analysis is required of this preliminary work, to provide a clearer indication of model performance. Use of additional performance metrics, including Pearson's correlation coefficient, would be applicable. Comparison against a benchmark from other research using a regression technique or simultaneous and proportional control of multiple DOFs is also required.

\section{CONCLUSION}

The experiment performed here shows it is feasible to use an LSTM neural network to perform prediction of wrist and finger movements with a good degree of accuracy. Combining the deep model with a feature engineering phase has proved advantageous. Investigating the replacement of this phase with a CNN for automated feature extraction is a pertinent next step. There is also still room for improvement with further work needed to optimize the LSTM model structure and hyperparameters, include additional performance measurements and more detailed analysis, and compare results with related research.

\section{REFERENCES}

[1] Nazmi, N. et al., "A Review of Classification Techniques of EMG Signals during Isotonic and Isometric Contractions," Sensors, 16(8), p.1304, 2016

[2] Amsuess, S. et al., "Context-Dependent Upper Limb Prosthesis Control for Natural and Robust Use," IEEE Trans Neural Syst Rehabil Eng., 24(7), pp.744-753, 2016.

[3] Mahmud, M. et al., "Applications of Deep Learning and Reinforcement Learning to Biological Data," IEEE Trans Neural Netw Learn Syst, 29(6), pp.2063-2079, 2018.

[4] Atzori, M., Cognolato, M. \& Müller, H., "Deep learning with convolutional neural networks applied to electromyography data: A resource for the classification of movements for prosthetic hands," Frontiers in Neurorobotics, 10(Sep), pp.1-10, 2016.

[5] Xia, P., Hu, J. \& Peng, Y., "EMG-Based Estimation of Limb Movement Using Deep Learning With Recurrent Convolutional Neural Networks," Artificial Organs, 42(5), pp.E67-E77, 2017.

[6] Wang, W. et al., "Sensor Fusion for Myoelectric Control Based on Deep Learning With Recurrent Convolutional Neural Networks." Artificial Organs, p.11, 2018.

[7] Vujaklija, I. et al., "Online mapping of EMG signals into kinematics by autoencoding," Journal of Neuro Engineering and Rehabilitation, $15(1)$, p.21, 2018.

[8] Atzori, M. et al. "Electromyography data for noninvasive naturally controlled robotic hand prostheses," Scientific data (2014), 1:140053, 2014.

[9] Elkoura, G. \& Singh, K.,. "Handrix: animating the human hand," in Proc. of ACM SIGGRAPH 2003 Symposium on Computer Animation. pp. 110-120, 2003.

[10] Castellini, C., Passig, G. \& Zarka, E., "Using ultrasound images of the forearm to predict finger positions." IEEE Trans Neural Syst Rehabil Eng, 20(6), pp.788-797, 2012.

[11] Robinson, C. et al. "Pattern classification of hand movements using time domain features of electromyography," in Proc. 4th Int. Conf. on Movement Computing, London, pp.1-6, 2017.

[12] Hudgins, B., Parker, P., \& Scott, R.N., "A New Strategy for Multifunction Myoelectric Control," IEEE Trans Biomed Eng, 40(1), pp.82-94, 1993.

[13] Hochreiter, S. \& Schmidhuber, J., "Long Short-Term Memory," Neural Computation, 9(8), pp.1735-1780, 1997.

[14] Deng, H., Zhang, L. \& Shu, X., "Feature memory-based deep recurrent neural network for language modeling," Applied Soft Computing Journal, 68, pp.432-446, 2018.

[15] Tan, J.H. et al., "Application of stacked convolutional and long shortterm memory network for accurate identification of CAD ECG signals," Computers in Biology and Medicine, 94(Dec 2017), pp.19-26, 2018. 